\title{
THE LÉVY-BAXTER THEOREM FOR GAUSSIAN RANDOM FIELDS: \\ A SUFFICIENT CONDITION
}

\section{TAKAYUKI KAWADA}

ABSTRACT. For a function $F$ of some class and for a Gaussian random field $\left\{X(\mathrm{t}) ; \mathrm{t} \in[0,1]^{N}\right\}, F$-variation $V_{F, n}(X)$ is defined as $V_{F, n}(X)=$ $\Sigma_{\mathbf{i}} F\left(X_{i, n}\right)$, where $X_{i, n}=\Delta_{N}^{s} \cdots \Delta_{1}^{s} X(s i), n=2^{-n}$, and $\Delta_{k}^{s}$ is the difference operator in the $k$ th component of $\mathbf{i}=\left(i_{1}, \ldots, i_{N}\right),\left(1 \leq i_{k} \leq 2^{n} ; k=1, \ldots\right.$, , $N)$. Here is presented a sufficient condition for the existence with probability 1 of the limit of the normalization of $V_{F, n}(X)$ as $n \rightarrow \infty$.

1. Introduction. Let $\left\{X(\mathbf{t}, \omega) ; t=\left(t_{1}, \ldots, t_{N}\right) \in[0,1]^{N}\right\}$ be a real, stochastically continuous Gaussian random field, based on a probability space $(\Omega, \Re, P), \omega \in \Omega$, with

$$
E(X(\mathbf{t}))=0, \quad E\left(\left(\Delta_{N}^{s} \cdots \Delta_{1}^{s} X(\mathrm{t})\right)^{2}\right)>0 \text { for any positive } s,
$$

where

$$
\Delta_{k}^{s} X(\mathrm{t})=X\left(t_{1}, \ldots, t_{k}, \ldots, t_{N}\right)-X\left(t_{1}, \ldots, t_{k}-s, \ldots, t_{N}\right), k=1,2, \ldots, N \text {. }
$$

Here, $\Delta_{N}^{s} \cdots \Delta_{1}^{s} X(\mathbf{t})$ is called a mixed increment.

Divide the unit cube $[0,1]^{N}$ into small cells by equipartition: Take $d_{n}=2^{n}$ and divide each unit interval $[0,1]$ by $d_{n}$ in each coordinate. Let us denote a cell by $c(\mathrm{i}, n)$, that is, for $\mathrm{i}=\left(i_{1}, \ldots, i_{N}\right), 1 \leq i_{k} \leq d_{n}(k=$ $1,2, \ldots, N)$,

$$
c(\mathbf{i}, n)=\left\{\mathbf{x}=\left(x_{1}, \ldots, x_{N}\right) ;\left(i_{k}-1\right) / d_{n} \leq x_{k} \leq i_{k} / d_{n}, k=1,2, \ldots, N\right\} .
$$

When two cells $c(\mathrm{i}, n)$ and $c(\mathrm{j}, n)$ are mutually component-wise disjoint, it is denoted by $c(\mathrm{i}, n) \cap c(\mathrm{j}, n)=\varnothing$. Let us denote by $X_{\mathrm{i}, n}$ the mixed increment over a cell $c(i, n)$, that is,

$$
X_{\mathbf{i}, n}=\Delta_{N}^{s} \ldots \Delta_{1}^{s} X(s \cdot \mathbf{i}), \quad s=1 / d_{n} .
$$

The above condition in this case reads $E\left(X_{\mathbf{i}, n}^{2}\right)>0$ for any $n$, and set $\sigma_{\mathbf{i}, n}^{2}$ $=E\left(X_{i, n}^{2}\right)$. Let us consider a class of functions satisfying the following conditions:

(a) $F(x)$ is positive and $F(0)=0$;

(b) for $l=(0,1 / 2]$,

Received by the editors July 1, 1974 and, in revised form, October 22, 1974. AMS (MOS) subject classifications (1970). Primary 60G15, 60F99.

Key words and phrases. Gaussian random field, mixed-increment, $F$-variation, strong limit of $F\lrcorner$ variation. 


$$
\inf _{\sigma \in I} \int_{-\infty}^{\infty} \frac{F(\sigma x)}{F(\sigma)} \exp \left(-x^{2} / 2\right) d x \neq 0 ;
$$

(c) for $k=0,1,2$,

$$
\sup _{\sigma \in I} \int_{-\infty}^{\infty}\left\{\frac{D^{k} F(\sigma x)}{F(\sigma)}\right\}^{2} \exp \left(-x^{2} / 2\right) d x<\infty,
$$

where $D^{k}=\partial^{k} / \partial x^{k}$;

(d) for $k=0,1$, and for every integer $m$,

$$
\left|D^{k} F(x)\right|=o\left(\exp \left(x^{2} / 2\right) / x^{m}\right), \text { as } x \rightarrow \infty \text {. }
$$

The forms of the integrands in the above suggest to us that some regular varying functions in the sense of Karamata may be included in this class. In fact, we see:

Examples of $F$.

and

$$
F(x)=|x|^{\rho}|L(|x|)| \quad(\rho \geq 2)
$$

$$
L(x)=\left\{\begin{array}{l}
1, \text { or } \\
(\log x)^{2 \mu_{1}} \ldots\left(\log _{(k)} x\right)^{2 \mu_{k}} \quad\left(\mu_{1}>1, \ldots, \mu_{k}>1\right), \quad \text { or } \\
\left(1+1 / x^{2}\right) \quad(\rho>\mu+2, \mu>0),
\end{array}\right.
$$

where $\log _{(k)}$ is the $k$-fold iteration of the logarithm.

Take a function $F$ from the above class and define $F$-variation of the random field $X$ by

and normalize

$$
V_{F, n}(X)=\sum_{\mathbf{i} \text { in all cells }} F\left(X_{\mathbf{i}, n}\right),
$$

$$
W_{F, n}(X)=V_{F, n}(X) / E\left\{V_{F, n}(X)\right\}
$$

Then we have

Theorem. Let $F$ satisfy the conditions (a) $\sim(\mathrm{d})$. If there exist monotone increasing sequences $\left\{G_{n} ; G_{n}\lceil\infty\right.$ as $n \uparrow \infty\}$ and $\left\{g_{n} ; g_{n}=o\left(d_{n}\right)\right.$ as $n \uparrow \infty\}$ such that

$$
\sum_{n}\left\{\frac{G_{n} \max _{\mathbf{i}} F\left(\sigma_{\mathbf{i}_{0} n}\right)}{\sqrt{g_{n}} \min _{\mathbf{i}} F\left(\sigma_{\mathbf{i}, n}\right)}\right\}^{2}<\infty
$$

$$
\frac{d_{n}^{2 N}\left(\min _{\mathbf{i}} \sigma_{\mathbf{i}, n}\right)^{2}}{g_{n}}
$$

$$
=O\left\{\max _{\substack{\mathbf{t} \in c(\mathbf{i}, n), \mathbf{s} \in c(\mathbf{j}, n) \\ c(\mathbf{i}, n) \cap \mathbf{n}(\mathbf{j}, n)=\varnothing}} \frac{\partial^{2 N}}{\partial t_{1} \cdots \partial t_{N} \partial s_{1} \cdots \partial s_{N}} E(X(\mathbf{t}) X(\mathbf{s}))\right\},
$$

as $n \rightarrow \infty$, then $P\left\{\lim _{n \rightarrow \infty} W_{F, n}(X)=1\right\}=1$ holds. 
S. M. Berman [2] initiated this type of problem in the case that $X(t)$ is Lévy's Brownian motion with $N$-parameters and $F(x)=|x|^{2 N}$. Recently, T. V. Arak [1] studied the same problem as the present and obtained a sufficient condition by a method different from ours.

2. Lemma. The following is required in the proof of the Theorem.

Lemma. Let $F(x)$ satisfy (c) and (d) in $\$ 1$. Let $(X, Y)$ be a bivariate normal random variable such that $E(X)=0, E\left(X^{2}\right)=\sigma_{1}^{2} ; E(Y)=0, E\left(Y^{2}\right)=\sigma_{2}^{2}$; and $E(X Y)=\rho \sigma_{1} \sigma_{2}$. Then

bolds.

$$
|E\{F(X) F(Y)\}-E\{F(X)\} E\{F(Y)\}| \leq \text { Const }|\rho| F\left(\sigma_{1}\right) F\left(\sigma_{2}\right)
$$

Proof. Let us denote by $\Phi^{(j)}(x)$ the $j$ th derivative of $\Phi(x) \equiv$ $\int_{-\infty}^{x} \exp \left(-u^{2} / 2\right) d u / \sqrt{2 \pi}$. We have a relation in terms of Hermite polynomials $\left\{H_{j}(x) ; j=0,1,2, \ldots\right\}$ :

$$
\Phi^{(j)}(x)=(-1)^{j-1} H_{(j-1)}(x) \cdot \exp \left(-x^{2} / 2\right) \quad(j=1,2, \ldots) .
$$

By means of Charlier's expansion, the bivariate normal density $\varphi(x, y ; \rho)$ in the Lemma is expanded as follows:

$$
\boldsymbol{\varphi}(x, y ; \rho)=\sum_{j=0}^{\infty} \frac{\rho^{j}}{j !} \Phi^{(j+1)}(x) \Phi^{(j+1)}(y) .
$$

Hence, we have, by inverting the order of integration and summation, $E\{F(X) F(Y)\}-E\{F(X)\} \cdot E\{F(Y)\}$

$$
\begin{aligned}
= & \frac{\rho}{\sigma_{1} \sigma_{2}} \int_{-\infty}^{\infty} F(x) \Phi^{(2)}\left(\frac{x}{\sigma_{1}}\right) d x \cdot \int_{-\infty}^{\infty} F(y) \Phi^{(2)}\left(\frac{y}{\sigma_{2}}\right) d y \\
& +\frac{1}{\sigma_{1} \sigma_{2}} \sum_{j=2}^{\infty} \frac{\rho^{j}}{j !} \int_{-\infty}^{\infty} F(x) \Phi^{(j+1)}\left(\frac{x}{\sigma_{1}}\right) d x \cdot \int_{-\infty}^{\infty} F\left(y^{\prime} \Phi^{(j+1)}\left(\frac{y}{\sigma_{2}}\right) d y .\right.
\end{aligned}
$$

In the second term, we have, by taking integration by parts twice and using (d)

$$
\frac{1}{\sigma_{1}} \int_{-\infty}^{\infty} F(x) \Phi^{(j+1)}\left(x / \sigma_{1}\right) d x=\int_{-\infty}^{\infty}\left(D^{2} F\left(\sigma_{1} x\right)\right) \Phi^{(j-1)}(x) d x,
$$

and, moreover, by Schwarz's inequality and (2.1),

$$
\begin{aligned}
& \leq \sqrt{(j-2) !}\left\{\int_{-\infty}^{\infty}\left(D^{2} F\left(\sigma_{1} x\right)\right)^{2} \exp \left(\frac{-x^{2}}{2}\right) d x\right\}^{1 / 2} \\
& =F\left(\sigma_{1}\right) \sqrt{(j-2) !}\left\{\int_{-\infty}^{\infty}\left[\frac{D^{2} F\left(\sigma_{1} x\right)}{F\left(\sigma_{1}\right)}\right]^{2} \cdot \exp \left(\frac{-x^{2}}{2}\right) d x\right\}^{1 / 2} \\
& \leq C \cdot F\left(\sigma_{1}\right) \sqrt{(j-2) !} \quad(\text { by }(\mathrm{c}))^{1}
\end{aligned}
$$

${ }^{1}$ Several absolute constants in the text will be denoted only by the capital $C$ without any suffix. 
Therefore, the second term is majorized by

$$
C \cdot F\left(\sigma_{1}\right) F\left(\sigma_{2}\right) \sum_{j=2}^{\infty} \frac{|\rho|}{j !}(j-2) ! .
$$

The first term is estimated similarly by virtue of (c) and (d) after an integration by parts. These complete the proof of the Lemma.

3. Proof of the Theorem. Since we have, by Tchebyshev's inequality,

$$
P\left\{\left|W_{F, n}(X)-1\right|>1 / G_{n}\right\} \leq G_{n}^{2} \cdot \operatorname{Var}\left(W_{F, n}(X)\right),
$$

it is sufficient to show

$$
\sum_{n} G_{n}^{2} \operatorname{Var}\left(W_{F, n}(X)\right)<\infty .
$$

For $\operatorname{Var}\left(W_{F, n}(X)\right)=\operatorname{Var}\left(V_{F, n}(X)\right) / E^{2}\left(V_{F, n}(X)\right)$, we have a first estimate

$$
E^{2}\left(V_{F, n}(X)\right) \geq C \cdot d_{n}^{2 N}\left(\min _{\mathrm{i}} F\left(\sigma_{i, n}\right)\right)^{2} .
$$

In fact,

$$
\begin{aligned}
E\left(V_{F, n}(X)\right) & \geq d_{n}^{N}\left(\min _{\mathbf{i}} E\left(F\left(X_{\mathbf{i}, n}\right)\right)\right) \\
& \geq d_{n}^{N} \min _{\mathbf{i}}\left\{F\left(\sigma_{\mathbf{i}, n}\right) \int_{-\infty}^{\infty} \frac{F\left(\sigma_{\mathbf{i}, n} x\right)}{F\left(\sigma_{\mathbf{i}, n}\right)} \exp \left(\frac{-x^{2}}{2}\right) d x / \sqrt{2 \pi}\right\} \\
& \geq C d_{n}^{N}\left(\min _{\mathbf{i}} F\left(\sigma_{\mathbf{i}, n}\right)\right) \quad(\text { by }(\mathrm{b})) .
\end{aligned}
$$

Next, $\operatorname{Var}\left(V_{F, n}(X)\right)$ is rewritten

$$
\operatorname{Var}\left(V_{F, n}(X)\right)=\sum_{\mathbf{i}, \mathbf{j}}\left\{E\left(F\left(X_{\mathbf{i}, n}\right) F\left(X_{\mathbf{i}, n}\right)\right)-E\left(F\left(X_{\mathbf{i}, n}\right)\right) E\left(F\left(X_{\mathbf{i}, n}\right)\right)\right\}
$$

which we split into two classes,

$$
=\sum_{(\mathrm{A})} v(\mathbf{i}, \mathbf{j})+\sum_{(\mathrm{B})} v(\mathbf{i}, \mathbf{j}),
$$

where the summation $(A)$ is restricted to the pair $(i, j)$, satisfying $c(i, n) \cap$ $c(\mathbf{j}, n)=\varnothing,(\mathrm{B})$ is for another pair, and $v(\mathbf{i}, \mathbf{j})$ is the summand.

As a result, (3.1) will be established if both

$$
\sum_{n} \sum_{(\mathrm{A})} \frac{G_{n}^{2} v(\mathrm{i}, \mathrm{j})}{d_{n}^{2 N}\left(\min _{\mathbf{i}} F\left(\sigma_{\mathrm{i}, n}\right)\right)^{2}}<\infty
$$

and

$$
\sum_{n} \sum_{(\mathrm{B})} \frac{G_{n}^{2} v(\mathrm{i}, \mathrm{j})}{d_{n}^{2 N}\left(\min _{\mathrm{i}} F\left(\sigma_{\mathrm{i}, n}\right)^{2}\right.}<\infty
$$

are verified. 
To verify (3.4). Since, by positivity of $F$, we have $v(i, j) \leq$ $E\left(F\left(X_{\mathbf{i}, n}\right) F\left(X_{\mathbf{j}_{\imath}, n}\right)\right)$, the first summation $\mathrm{S}_{(\mathrm{B})}(n)$ in (3.4) is estimated as follows:

$$
\mathrm{S}_{(\mathrm{B})}(n) \leq \sum_{(\mathrm{B})} \frac{G_{n}^{2} E\left(F\left(X_{\mathbf{i}, n}\right) F\left(X_{\mathbf{i}, n}\right)\right)}{d_{n}^{2 N}\left(\min _{\mathbf{i}} F\left(\sigma_{\mathbf{i}, n}\right)\right)^{2}} \leq \#\{(\mathbf{i}, \mathbf{j}) \text { in (B) }\} \frac{G_{n}^{2}\left(\max _{\mathbf{i}} E\left(F^{2}\left(X_{\mathbf{i}, n}\right)\right)\right)}{d_{n}^{2 N}\left(\min _{\mathbf{i}} F\left(\sigma_{\mathbf{i}, n}\right)\right)^{2}}
$$

where $\#(Z)$ is the cardinal number of the set $Z$. Since, by (c), $E\left(F^{2}\left(X_{i, n}\right)\right)=F^{2}\left(\sigma_{i, n}\right) \int_{-\infty}^{\infty}\left[\frac{F\left(\sigma_{i, n} x\right)}{F\left(\sigma_{i, n}\right)}\right]^{2} \exp \left(\frac{-x^{2}}{2}\right) d x / \sqrt{2 \pi} \leq C \cdot F^{2}\left(\sigma_{i, n}\right)$, and $\#\{(\mathbf{i}, \mathbf{j})$ in $(\mathrm{B})\} \leq d_{n}^{2} N-1$, we have

$$
\mathrm{S}_{(\mathrm{B})}(n) \leq C \cdot G_{n}^{2}\left\{\frac{\max _{\mathbf{i}} F\left(\sigma_{\mathrm{i}, n}\right)}{\min _{\mathbf{i}} F\left(\sigma_{i, n}\right)}\right\}^{2} / d_{n} \leq C \cdot\left\{\frac{G_{n} \max _{\mathrm{i}} F\left(\sigma_{\mathrm{i}, n}\right)}{\sqrt{g_{n}} \min _{\mathbf{i}} F\left(\sigma_{i, n}\right)}\right\}^{2} g_{n} / d_{n} .
$$

Therefore, by (1.1), we have $\Sigma_{n} \mathrm{~S}_{(\mathrm{B})}(n)<\infty$.

To verify (3.3). The Lemma is applied to $v(\mathbf{i}, \mathbf{j})$ in the first sum $\mathrm{S}_{(A)}(n)$ in (3.3) to obtain the estimate

$$
\mathrm{S}_{(\mathrm{A})}(n) \leq C \cdot G_{n}^{2} d_{n}^{2 N} \frac{\max _{\mathbf{i}, \mathbf{j}}\left\{\rho_{\mathbf{i}, \mathbf{j}} F\left(\sigma_{\mathbf{i}, n}\right) F\left(\sigma_{\mathbf{j}, n}\right)\right\}}{d_{n}^{2 N}\left\{\min _{\mathbf{i}} F\left(\sigma_{\mathbf{i}, n}\right)\right\}^{2}} .
$$

Further, by virtue of the relation in the case $c(\mathbf{i}, n) \cap c(\mathbf{j}, n)=\varnothing$,

$$
\sigma_{\mathbf{i}, n} \sigma_{\mathbf{i}, n} \rho_{\mathbf{i}, \mathbf{j}}=E\left(X_{\mathbf{i}, n} X_{\mathbf{j}, n}\right)=\iint_{c(\mathbf{i}, n) \times c(\mathbf{j}, n)} \frac{\partial^{2 N}}{\partial t_{1} \cdots \partial s_{N}} E(X(\mathbf{t}) X(\mathbf{s})) d \mathbf{t} d \mathbf{s},
$$

we have a majorization as follows:

$$
\begin{aligned}
& \mathrm{S}_{(\mathrm{A})}(n) \leq C \cdot\left\{\frac{G_{n} \max _{\mathbf{i}} F\left(\sigma_{\mathbf{i}_{,} n}\right)}{\sqrt{g_{n}} \min _{\mathbf{i}} F\left(\sigma_{\mathbf{i}_{9}, n}\right)}\right\}^{2} \\
& \cdot\left\{\max _{\substack{\mathrm{t} \in c(\mathrm{i}, n), \mathrm{s} \in c(\mathrm{j}, n) \\
c(\mathrm{i}, n) \text { ก } c(\mathrm{j}, n)=\varnothing}} \frac{\partial^{2 N}}{\partial t_{1} \ldots \partial s_{N}} E(X(\mathrm{t}) X(\mathrm{~s}))\right\} /\left\{\frac{d_{n}^{2 N}\left(\min _{\mathrm{i}} \sigma_{\mathrm{i}, n}^{2}\right)}{g_{n}}\right\} .
\end{aligned}
$$

Hence, we have, by (1.1) and (1.2), $\Sigma_{n} \mathrm{~S}_{(\mathrm{A})}(n)<\infty$. Thus the Theorem is established.

4. Examples. (4.A) Consider the Ornste in-Uhlenbeck random field $\left\{U(\mathrm{t}) ; \mathrm{t} \in[0,1]^{N}\right\}:$

$U(\mathrm{t})=\alpha \int_{0}^{\mathrm{t}} \exp \left(-\beta \sum_{i=1}^{N}\left(t_{i}-\tau_{i}\right)\right) d B(\boldsymbol{r}) \quad(\alpha>0, \beta>0) \quad \mathrm{t}=\left(t_{1}, \ldots, t_{N}\right)$, where $B(t)$ is the $N$-parameter Wiener field with

$$
E(B(\mathrm{t}))=0, \quad E(B(\mathrm{t}) B(\mathrm{~s}))=\prod_{i=1}^{N} \min \left(t_{i}, s_{i}\right) .
$$


The mean and the correlation of $U(\mathrm{t})$ are

$$
E(U(\mathbf{t}))=0, \quad E(U(\mathbf{t}) U(\mathbf{s}))=\alpha^{2} \cdot \exp \left(-\beta \sum_{i=1}^{N}\left|t_{i}-s_{i}\right|\right) .
$$

Set $d_{n}=2^{n}$ and $F(x)=|x|^{2 \lambda},(\lambda>1$, real $)$. The variance of the mixed increment $U_{i, n}$ is

$$
E\left(U_{i, n}^{2}\right)=2^{N} \alpha^{2}\left(1-\exp \left(-\beta 2^{-n}\right)\right)^{N}
$$

for any i. Then we have

$$
P\left\{\lim _{n \rightarrow \infty} \frac{2^{n \lambda}}{2^{n N}} V_{F, n}(U)=\left(2^{N+1} \alpha^{2} \beta^{N}\right)^{\lambda} \Gamma(\lambda) / \sqrt{2 \pi}\right\}=1 .
$$

(4.B) Consider the Gaussian field $\left\{X(t) ; t \in[0,1]^{N}\right\}$ with

$$
E(X(\mathbf{t}))=0, \quad E(X(\mathbf{t}) X(\mathbf{s}))=\left(|\mathbf{t}|^{\alpha}+|\mathbf{s}|^{\alpha}-|\mathbf{t}-\mathbf{s}|^{\alpha}\right) / 2 \quad(0<\alpha<2) .
$$

Set $d_{n}=2^{n}$ and $F(x)=|x|^{2 \lambda}(|\log | x||)^{2 k},(\lambda>1$, real; $k>1$, integer $)$. Observing that

where

$$
E\left(V_{F, n}(X)\right)=2^{n N}\left(2^{-n a} \sigma_{1}^{2}\right) \cdot \sum_{i=0}^{2 k}\left(\begin{array}{c}
2 k \\
i
\end{array}\right)\left(\log 2^{-n a / 2} \sigma_{1}\right)^{i} I(i),
$$

$$
\sigma_{1}^{2}=E\left\{\left(\Delta_{N}^{1} \cdots \Delta_{1}^{1} X(1)\right)^{2}\right\}=(2 N) ! 2^{N-1} \sum_{p=1}^{N}(-1)^{p-1} p^{a / 2}\left(\begin{array}{l}
N \\
p
\end{array}\right) / N ! 2^{N}
$$

(cf. [3]), and

$$
I(i)=\int_{-\infty}^{\infty}|x|^{2 \lambda}(|\log | x||)^{2 k-1} \exp \left(\frac{-x^{2}}{2}\right) d x / \sqrt{2 \pi},
$$

we have

$$
P\left\{\lim _{n \rightarrow \infty} \frac{2^{n a \lambda}}{n^{2 k} 2^{n N}} V_{F, n}(X)=\sigma_{1}^{\lambda+2 k}((\alpha \log 2) / 2)^{2 k} I(2 k)\right\}=1 .
$$

(4.C) Consider the Gaussian field $\left\{X(t) ; t \in[0,1]^{N}\right\}$ with

$$
E(X(\mathrm{t}))=0, \quad E(X(\mathrm{t}) X(\mathrm{~s}))=\prod_{i=1}^{N}\left\{\min \left(\tau_{i}\left(t_{i}\right), \tau_{i}\left(s_{i}\right)\right)\right\},
$$

where $\tau_{i}(x),(i=1,2, \ldots, N)$ is a monotone-increasing $C^{1}$-function such that $d \tau_{i}(x) / d x=O(1)$ as $x \rightarrow 0$. Define a stochastic integral for a continuous function $f$ over the cube $B=[0,1]^{N}$ :

$$
Y(\mathbf{t})=\int_{B}\left(\operatorname{Ind}\left(\prod_{i=1}^{N}\left[0, t_{i}\right]\right) f\right)(\mathbf{u}) d X(\mathbf{u}), \quad \mathbf{t}=\left(t_{1}, \ldots, t_{N}\right),
$$

where $\operatorname{Ind}(A)\left(A\right.$ is a set in $\left.R^{N}\right)$ is the indicator-function of the set $A$.

We observe, for $d_{n}=2^{n}$,

$$
E(Y(\mathbf{t}))=0, \quad E(Y(\mathrm{t}) Y(\mathbf{s}))=\int_{[0,1]^{N}}\left(\operatorname{Ind}\left(\prod_{i=1}^{N}\left[0, \min \left(t_{i}, s_{i}\right)\right]\right) f^{2}\right)(\mathbf{u}) d \boldsymbol{r}(\mathbf{u}),
$$

where $d r(\mathbf{u})=\mathrm{II}_{i=1}^{N} d \tau_{i}\left(u_{i}\right)$, and observe, for an increment $Y_{\mathbf{i}, n}$, 


$$
E\left(Y_{\mathbf{i}, n}^{2}\right)=\int_{[0,1]^{N}}\left(\operatorname{Ind}\left(\prod_{k=1}^{N}\left[\frac{i_{k}-1}{2^{n}}, \frac{i_{k}}{2^{n}}\right]\right) f^{2}\right)(\mathbf{u}) d \tau(\mathbf{u}),
$$

for $\mathbf{i}=\left(i_{1}, \ldots, i_{N}\right)$. This field has not necessarily homogeneous mixed increments. When $f(\mathbf{u})>c\left(c\right.$ a positive constant) and $F(x)=|x|^{2 \lambda}$, $\max _{\mathbf{i}} F\left(\sigma_{\mathbf{i}, n}\right) / \min _{\mathbf{i}} F\left(\sigma_{\mathbf{i}, n}\right)$ is majorized by an absolute constant. Then we have

$$
P\left\{\lim _{n \rightarrow \infty} \frac{2^{n N \lambda}}{2^{n N}} V_{F, n}(Y)=K \int_{[0,1]^{N}} f^{2 \lambda}(\mathbf{u})\left(\prod_{i=1}^{N} \tau_{i}^{\prime}\left(u_{i}\right)\right)^{\lambda} d \mathbf{u}\right\}=1,
$$

where $K=2^{\lambda} \cdot \Gamma(\lambda+1 / 2) / \sqrt{\pi}$ and $\tau_{i}^{0}\left(u_{i}\right),(i=1, \ldots, N)$ is the derivative of $\tau_{i}(x)$ at $x=u_{i}$

Remark. In Example (4.A), $U(t)$ is also defined from $B(t)$ similarly in the case of one dimension by the following transform of "time":

$$
\alpha B\left(e^{2 \beta t} 1, e^{2 \beta t_{2}}, \ldots, e^{2 B t} N\right)=\left(\prod_{i=1}^{N} e^{2 \beta t_{i}}\right)^{1 / 2} U\left(t_{1}, \ldots, t_{N}\right)
$$

\section{REFERENCES}

1. T. V. Arak, On the Lévy-Baxter theorems for random fields, Teor. Verojatnost. i Primenen. 17 (1972), 153-160. (Russian) MR 45 \#7798.

2. S. M. Berman, A version of the Lévy-Baxter theorem for the increments of Brownian motion of several parameters, Proc. Amer. Math. Soc. 18 (1967), 1051-1055. MR $36 \# 6008$.

3. P. T. Strait, On Berman's version of the Lévy-Baxter theorem, Proc. Amer. Math. Soc. 23 (1969), 91-93. MR 39 \#7662.

KOBE COLLEGE OF COMMERCE, TARUMI, KOBE (655), JAPAN 